\title{
A Time Serious Model of American Development Based on Data Mining Technology
}

\author{
Sheng Guan ${ }^{1, a^{*}}$, Lingxiao Yang ${ }^{1, b}$ and Ming Yang ${ }^{1, c}$ \\ ${ }^{1}$ Department of Information Management, Dalian Neusoft University of Information, China \\ aguansheng@neusoft.edu.cn, ${ }^{\text {b} 1785653504 @ q q . c o m, ~ ' 626841452 @ q q . c o m ~}$ \\ * please mark the corresponding author with an asterisk
}

Keywords: Economic energy indicator; Data mining; Time series model; American development

\begin{abstract}
As we all know, energy production is an indispensable part of the national economic structure. In this paper, we based on data mining technology to monitor and analysis data by cumulative data on energy production and consumption. We do data analysis to the objective function by correlation. We analyze its correlation with changes in economic indicator by taking energy consumption as the dependent variable. It is supporting for proposed energy solutions.
\end{abstract}

\section{Introduction}

Energy production and use are both important parts of any economy. In the United States, many aspects of energy policy are scattered at the national level. In addition, industries in different geographical regions and different countries have an impact on energy use and production[1]. In 1970, the western Interstate Energy Agreements were formed in the United States in the 12 western states and its mission was mainly to promote cooperation in these countries in the development and management of nuclear energy technologies[2]. Interstate contracts are contractual arrangements between two or more countries that agree on specific policy issues and adopt a set of standards or cooperate with one another on a particular regional or national issue[3,4].

\section{The optimal model of economic indicators}

Because much of the data is collected interstate and territorial during the data collection process, the data collected has its own regulations in each state and has been enforced at some point in the past. As a result, each state collects information differently and at the same time it starts collecting information. Production energy and energy consumption within a state are separated, some of the data on energy production is missing and data mining can not be done directly. Before the model is established, the data is preprocessed, the redundant data and the noise data are removed, the format of the data is adjusted, and the mining is conveinent.

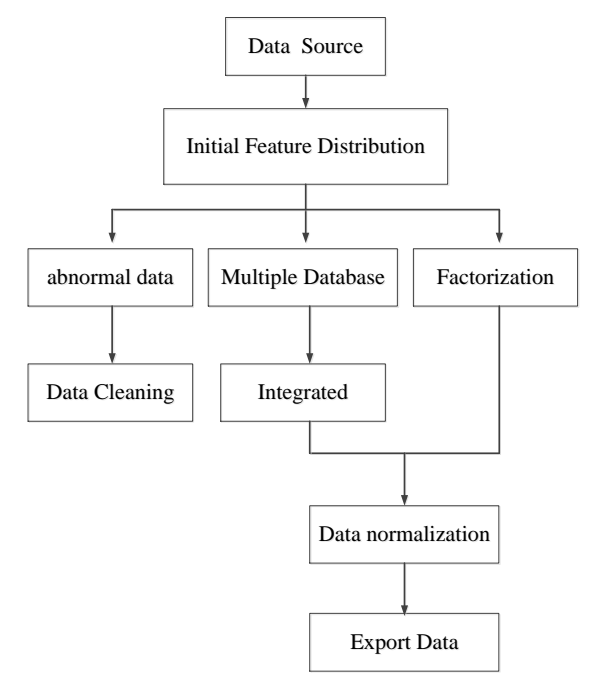

Figure 1. Finite Data preprocessing flow chart 
The processed data into the Oracle database, data preprocessing process shown in Figure one. To solve problem of energy allocation and evaluation, we explore how the economic indicators will change according to different factors. We establish the multi-objective programming model, take the energy consumption as the dependent variable and the univariate correlation of the objective function Data analysis, the establishment of the most economic indicators of the model, the amount of energy contributed by energy activities as an independent variable, analysis of changes in economic indicators and relevance. The energy allocation model for the states is as shown.

$$
\begin{aligned}
& \mathrm{Z}=\sum_{i=1}^{n} F_{n}(x)=\left[\sum_{i=1}^{n} \max (\min ) f_{n}(x)\right] \\
& \mu(x)=\left[\sum_{i=1}^{n} \varphi_{n}(x) \leq G=\left[\begin{array}{c}
g_{1} \\
g_{2} \\
\vdots \\
g_{n}
\end{array}\right]\right] \\
& \max \varphi=\sum_{i=1}^{k} \varphi_{i} \gamma_{i} \\
& \min \sum_{i=1}^{n} a_{i} x_{i} \\
& \varphi_{i}=\left(X_{1}, X_{2}, X_{3} \cdots X_{n}\right) \leq g_{i}(i=1 \cdots m) \\
& f_{i}+d_{i}^{-}-d_{i}^{+}=f_{i}^{+}(i=1 \cdots k)
\end{aligned}
$$

Where:

$X_{i}$ : The amount of energy contributed by the ith energy activity.

$a_{i}$ : The amount of energy spent on the ith clock.

$F_{n}:$ The objective function of the energy activity of the ith clock

$\lambda_{i}$ : The weight of each energy indicator.

\section{Time Series Prediction Model}

The Time Bench system provides a way for time series data to map data structures and data to time. In this paper, Time Bench system shadow economic indicators of the main factors for data visualization. Time Bench frame structure diagram as shown.

For data mining techniques based on feature parameters, the feature parameters may be used as an objective function to assess the degree to which the data objects belong to a central cluster[5]. If you delete a data object resulting in a significant improvement in the goal, that the data object can be identified as abnormal objects.

$$
p(x)=\prod_{j=1}^{n} p\left(x_{j} ; \mu_{j} ; \sigma_{j}^{2}\right)=\prod_{j=1}^{n} \frac{1}{\sqrt{2 \pi} \sigma_{j}} \exp \left(-\frac{\left(x_{j}-\mu_{j}\right)^{2}}{2 \sigma_{j}^{2}}\right)
$$

If $\mathrm{p}(\mathrm{x})<\varepsilon$, it is determined that the test data object is abnormal. The current transaction status mode is compared with the normal mode to detect whether the ATM system has an abnormal transaction status. Input classified "normal" and "abnormal" indicators in the data mining module[6]. According to the normal and abnormal patterns and rules in the database, the relative comparisons are compared by the pattern comparison algorithm to obtain the similarities. 


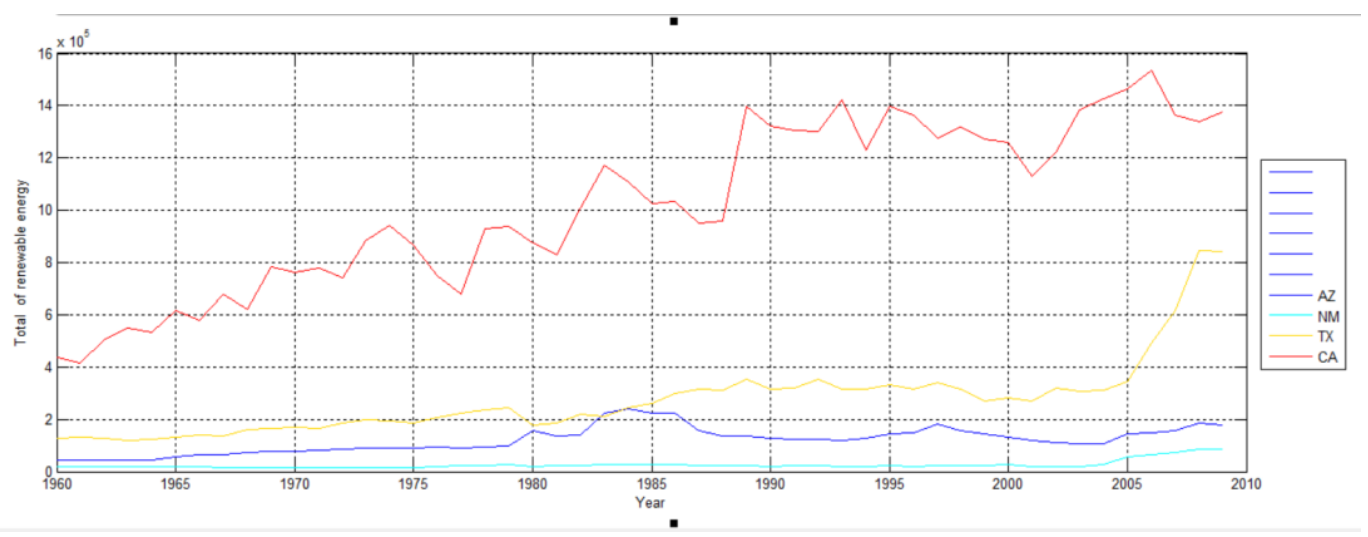

Figure 2. Finite Evaluating module

If the similarity is greater than the system-defined threshold, the data may be normal behavior, and then compare the data object with the abnormal patterns in the database for pattern comparison. If the similarity is less than System-defined threshold, you can conclude that the user behavior is normal behavior, if the similarity is greater than the specified threshold, may be marked as may belong to the abnormal data object.

\section{Data Detection Module}

Based on the optimal model of economic indicators above, the relationship between energy development-time and energy development-time, the historical data are summarized and imported into Oracle database[7]. The time series model based on exponential smoothing is predicted by MATLAB programming. The program runs Flow chart show.

For each state to adopt the same policy, each state needs to be integrated (geography, climate, etc.), select the appropriate renewable energy, and this question will be designed in the statistical distribution method under the framework of an exponential smoothing model based on data mining[8]. The extracted weights in part1 are used as indexes to detect the outlier of target data object, including three modules: data mining, anomaly detection and pattern updating.

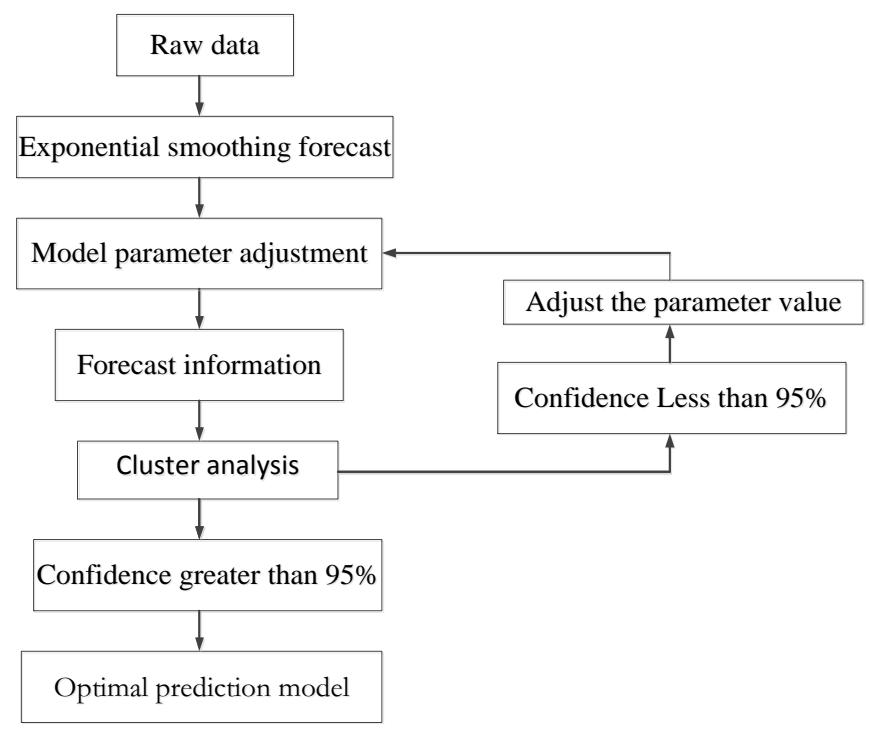

Figure 3. Finite Data detection module 


\section{Data Mining Module}

The data mining module takes characteristic parameters extracted and uses the index correlation analysis and the time series analysis algorithm to mine the basic information database to find the association rules and frequent sequence patterns[9]. Then, the frequent patterns excavated are classified, and the normal and abnormal behavior patterns of renewable energy based on the characteristic parameters are obtained.

\section{Anomaly Detection and Mode Update}

All data objects are clustered first, and then the contribution of the data objects to the cluster is evaluated[10]. For cluster classes generated based on feature parameters, the distance from the data object to its cluster center can be used to measure the extent to which the data object belongs to a cluster.

Module update module can constantly amend and update the normal mode of the system. Because in the real world data changes over time, the state of use of energy may exhibit some trend over time, rendering the current state of the trading state model no longer valid.

\section{Summary}

According to the problem solving problem, the model will be gradually established. After the basic economic indicators have been determined and tested, a new model will be set up. The hierarchy will be further promoted and understood easily.

\section{References}

[1] Harbor effect of energy-saving emission reduction evaluation system will have [J]. Water Transport Engineering, 2017, (z1): 36.

[2] Shi Yuanchun. Reflections on the development of clean and renewable energy sources [J]. Shaanxi Electric Power, 2014, (7): 1-3. DOI: 10.3969 / j.issn.1673-7598.2014.07.001

[3] Xiao Hongbo, Li Jun, Li Zongtai, et al.Analysis of rural energy consumption in Beijing and its influencing factors: A survey based on 1866 rural households in Beijing [J] .Journal of China Agricultural Resources and Regional Planning, 2017, (10) 137. DOI: 10.7621 / cjarrp.1005-9121.20171018.

[4] Li Zongtai, Li Hua, Xiao Hongbo, et al.Analysis on energy consumption structure and influence factors of rural life in Beijing [J] .Chinese Journal of Eco-Economics, 2017, (12): 101-104.

[5] Yezli S, Alotaibi B M, Saeed A A. The Hajj Health Requirements: time for a serious review[J]. Lancet, 2016, 387(10021):845-846.

[6] Schuklenk U. Time to Take Another Serious Look at Conscientious Objection.[J]. Bioethics, 2016, 30(9):655-656.

[7] Yan H, Zhan J, Yang H, et al. Long time-series spatiotemporal variations of NPP and water use efficiency in the Lower Heihe River Basin with serious water scarcity[J]. Physics \& Chemistry of the Earth Parts A/b/c, 2016, 96.

[8] Tang D, Fan H, Zhang Y. Review on Landsat Time Series Change Detection Methods[J]. Journal of Geo-Information Science, 2017.

[9] Omran T, Miquel M, Laurini R. Continuous data warehouse: concepts, challenges and potentials[J]. Proc. int.conf. on Geoinformatics, 2017.

[10] Samuel J. Towards a Data Warehouse Fed with Web Services[J]. 2016, 8465:874-884. 